\title{
Naloxone induces endoplasmic reticulum stress in PC12 cells
}

\author{
SOYOUNG SEO ${ }^{1}$, YOUNG-SOOK KWON ${ }^{2}$, KWEON YU $^{3}$, SEUNG-WHAN KIM ${ }^{4}$, \\ $\mathrm{O}-Y U_{\mathrm{KWON}}{ }^{5}$, KYUNG-HEE KANG ${ }^{6}$ and KISANG KWON ${ }^{7}$ \\ ${ }^{1}$ Inje University Busan-Paik Hospital, Busan 614-735; \\ ${ }^{2}$ Department of Nursing, JoongBu University, Geumsan 312-702; \\ ${ }^{3}$ Korea Research Institute of Bioscience \& Biotechnology, Daejeon 305-806; \\ ${ }^{4}$ Department of Emergency Medicine, Chungnam National University Hospital, Daejeon 301-721; \\ ${ }^{5}$ Department of Anatomy, College of Medicine, Chungnam National University, Daejeon 301-747; \\ ${ }^{6}$ Department of Dental Hygiene, Konyang University, Daejeon 301-832; \\ ${ }^{7}$ Department of Biomedical Laboratory Science, College of Health and Welfare, \\ Kyungwoon University, Gumi 730-739, Republic of Korea
}

Received June 10, 2013; Accepted January 29, 2014

DOI: $10.3892 / \mathrm{mmr} .2014 .1935$

\begin{abstract}
Naloxone is an opioid inverse agonist used in the treatment of opiate overdose, with well known pharmacology. In the present study, we determined the effects of naloxone on the unfolded protein response (UPR) in PC12 cells. Data from a 3-(4,5-dimethylthiazol-2-yl)-2,5-diphenyltetrazolium bromide (MTT) assay indicated that naloxone may accelerate PC12 cell apoptosis in a dose-dependent manner. We also demonstrated that naloxone upregulated gene expression of endoplasmic reticulum (ER) chaperones, including binding immunoglobulin protein (Bip), calnexin, ER protein 29 (ERp29) and protein disulfide isomerase (PDI), and ER stress sensors, including activating transcription factor 6 (ATF6), inositol-requiring enzyme 1 (IRE1) and protein kinaselike ER kinase (PERK). In addition, naloxone also induced typical ER stress phenomena, including ART6 proteolytic cleavage, eIF2 $\alpha$ phosphorylation and XBP1 mRNA splicing. Furthermore, naloxone upregulated gene expression of ER chaperones and ER stress sensors in in vivo experiments. To the best of our knowledge, these results are the first to indicate that naloxone induces ER stress in vitro and in vivo.
\end{abstract}

\section{Introduction}

Naloxone is an opioid inverse agonist, used to reverse the effects of narcotic drugs and also to counter the effects of opiate

Correspondence to: Professor Kisang Kwon, Department of Biomedical Laboratory Science, College of Health and Welfare, Kyungwoon University, Gumi, Gyeongbuk 730-739, Republic of Korea

E-mail: ppkisang@empas.com

Key words: naloxone, endoplasmic reticulum stress, endoplasmic reticulum chaperones overdose in the emergency department (1). It has a chemical structure that is similar to oxymorphone with the only difference being the substitution of the N-methyl group with an allyl group (Fig. 1A). The name naloxone is derived from $\mathrm{N}$-allyl and oxymorphone (2). Naloxone is most commonly injected intravenously for rapid action within a few minutes. However, the side effects of naloxone include headaches, sudden chest pain, vomiting and an irregular pulse, but the cause of these side effects is not yet fully understood at the molecular level. Limited experimental data indicated that naloxone is able to interfere with granulocytopoiesis in the bone marrow, inhibit macrophage activation and albumin secretion from liver cells, activate various transcriptional factors, attenuate the increase of heat-shock protein expression and is also associated with dopamine secretion (3-8).

The endoplasmic reticulum (ER) is an intracellular organelle found in each eukaryotic cell and its major biological function includes the post-translational modification of secretory proteins. The ER has a sophisticated signal transducing system that senses and responds to changes in cellular homeostasis (9). ER stress is induced by a UPR to the adaption and survival of cells and/or tissues by expression of ER chaperones, including binding immunoglobulin protein (Bip), calnexin, protein disulfide isomerase (PDI) and ER protein 29 (ERp29), which directly or indirectly mediate multiple molecular biological processes via ER stress sensors [inositol-requiring enzyme 1 (IRE1); protein kinase-like ER kinase (PERK) and activating transcription factor 6 (ATF6)]. The ER stress response in mammalian cells is triggered by the dissociation of Bip from stress transducers, including PERK, ATF6 and IRE1. Bip binds to ER luminal unfolded proteins and activates the ER stress response $(10,11)$.

Naloxone is known to evoke a series of biochemical events in cells (12). However, to date, a direct effect of naloxone on ER stress has not been demonstrated. The objective of the present study is to understand the effects of naloxone on cell survival and induction of apoptosis as measured by the 3-(4,5-dimethylthiazol-2-yl)-2,5-diphenyltetrazolium bromide 
(MTT) assay. The induced apoptosis was observed and the association with ER stress was examined. The results demonstrate that naloxone dose dependently induces gene expression of ER chaperones and ER stress sensors, respectively.

\section{Materials and methods}

Cell culture and naloxone exposure. The PC12 cell line derived from a pheochromocytoma of the rat adrenal medulla, is a useful model system for neuronal experiments. The cells were cultured on collagen-coated flasks in $85 \%$ RPMI-1640 supplemented with 25 mM HEPES buffer, $10 \%$ heat-inactivated horse serum, $5 \%$ heat-inactivated fetal bovine serum, $2 \mathrm{mM}$ L-glutamine, $1 \mathrm{mM}$ sodium pyruvate, $1 \mathrm{~g} / \mathrm{l} \mathrm{d}$-(+)-glucose and antibiotics: $25 \mu \mathrm{g} / \mathrm{ml}$ streptomycin and $25 \mathrm{U} / \mathrm{ml}$ penicillin. Cells were maintained in a humidified incubator at $37^{\circ} \mathrm{C}$ in a $5 \% \mathrm{CO}_{2}$ atmosphere. The medium was changed every $48 \mathrm{~h}$. Cells were rinsed with $1 \mathrm{X}$ PBS $\mathrm{pH} 7.0$ and detached with $0.25 \%$ trypsin/EDTA. Following centrifugation at $1,000 \mathrm{xg}$ for $5 \mathrm{~min}$, cells were subcultured in $25 \mathrm{~cm}^{2}$ flasks using a subcultivation ratio of $1: 2$ to $1: 4$ and images were captured every $24 \mathrm{~h}$ with an inverted microscope. Cells were passaged twice a week. The $80 \%$ confluent monolayer of PC12 cells was treated with naloxone at the indicated doses and times. Total RNA from cultured cells was extracted using an RNA isolation reagent (TRI-Reagent, Ambion, Austin, TX, USA) and used for the following RT-PCR experiments. The study was approved by the ethics committee of Chungnam National University (Deajeon, Republic of Korea)

Cell viability measurement by MTT assay. The cell growth and viability of PC12 cells were determined by an MTT assay, (Sigma-Aldrich, St. Louis, MO, USA). The cells were seeded in 96-well plates and treated with each flavonoid at the indicated concentration. Once cells were treated with flavonoids for the indicated times, the MTT solution was added to each well and the plates were incubated for an additional $4 \mathrm{~h}$ at $37^{\circ} \mathrm{C}$. Following removal of the medium, the formazan crystals were solubilized in DMSO. The color development was monitored at $595 \mathrm{~nm}$ with a reference wavelength of $650 \mathrm{~nm}$.

Semiquantitative RT-PCR. RT-PCR using the forward primer (F) (5'-ACCACCAGTCCATCGCCATT-3') and reverse primer (R) (5'-CCACCCTGGACGGAAGTTTG-3') for IRE1; F (5'-CTAGGCCTGGAGGCCAGGTT-3') and R (5'-ACCCTGGAGTATGCGGGTTT-3') for ATF6; F (5'-GGTCTGGTTCCTTGGTTTCA-3') and R (5'-TTCGCTGGCTGTGTAACTTG-3') for PERK; F (5'-AGTG GTGGCCACTAATGGAG-3') and R (5'-TCTTTTGTCAGG GGTCGTTC-3') for Bip; F (5'-GGGAGTCTTGTCGTG GAATTG-3') and R (5'-TGCTTTCCAAGACGGCAGA-3') for calnexin; F (5'-CAGGATTTGCCCTATCCAGA-3') and $\mathrm{R}$ (5'-GTCATTCCGTTCCTTCTCCA-3') for PDI; F (5'-TACAAGGTCATTCCCAAAAGCAAGT-3') and R (5'-CGGAAGAGGTAGAAGACTGGGTAGC-3') for ERp29; F (5'-ACATCAAATGGGGTGATGCT-3') and R (5'-AGGAGACAACCTGGTCCTCA-3') for $\beta$-actin. RT-PCR primers were supplied from Bioneer Co. (Taejon, Chungcheongnam, Republic of Korea). Unless otherwise noted,
A
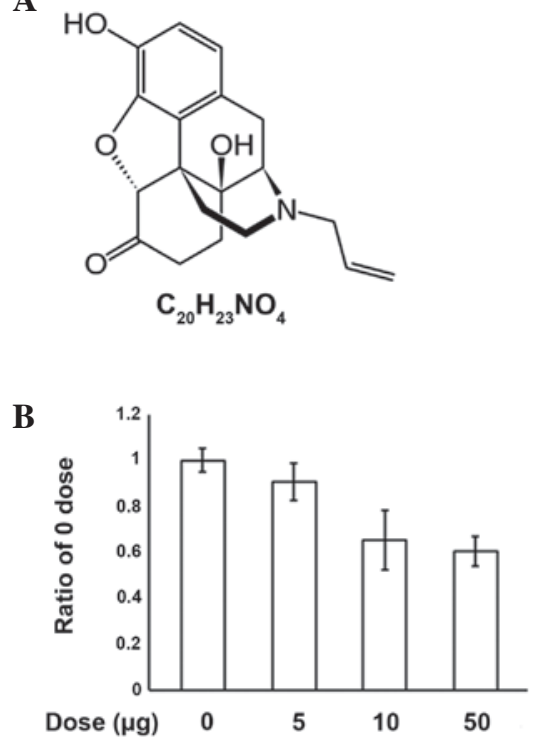

Figure 1. Effect of naloxone on inhibition of PC12 cell proliferation. (A) Chemical structure of naloxone. (B) Dose-dependent inhibition of cell proliferation. The cells were treated with the indicated concentrations of naloxone for $24 \mathrm{~h}$. Apoptotic cells were evaluated by MTT assay. The experiments were performed three times and results represent the average.

all chemicals were purchased from Sigma-Aldrich. RT-PCR conditions were for 30 cycles $\left(94^{\circ} \mathrm{C}\right.$ for $30 \mathrm{sec} ; 58^{\circ} \mathrm{C}$ for $30 \mathrm{sec}$; and $72^{\circ} \mathrm{C}$ for $1 \mathrm{~min}$ but $10 \mathrm{~min}$ in the final cycle) using the primers with Taq DNA polymerase.

Western blotting. Immunoblotting was performed according to the standard procedure. PC12 cells were scraped, lysed by the addition of SDS sample buffer $(62.5 \mathrm{mM}$ Tris- $\mathrm{HCl}$ $\mathrm{pH}$ 6.8, 6\% w/v SDS, 30\% glycerol, $125 \mathrm{mM}$ DTT, $0.03 \%$ w/v bromophenol blue) and separated by SDS-PAGE. The proteins were transferred onto a nitrocellulose membrane and the membrane was incubated with the primary antibodies overnight at $4^{\circ} \mathrm{C}$. The blots were developed using an enhanced chemiluminescence western blotting detection system kit (Amersham, Uppsala, Sweden). Rabbit anti-eIF2 $\alpha$ antibody, eIF2 $\alpha$-P antibody and goat anti-actin antibody were obtained from Santa Cruz Biotechnology, Inc. (Santa Cruz, CA, USA). Mouse anti-ATF6 antibody was obtained from Imgenex (San Diego, CA, USA).

\section{Results}

Induction of apoptosis. An MTT assay was performed to investigate the process of apoptosis induced by various concentrations of naloxone in PC12 cells (Fig. 1B). We observed that increasing concentrations of naloxone $(0,5,10$ and $50 \mu \mathrm{g} / \mathrm{ml})$ caused dose dependent increases in apoptosis, however, no specific morphological changes were observed (data not shown). The results demonstrate that naloxone induces apoptosis in PC12 cells in a dose-dependent manner.

Expression of ER chaperones and ER stress sensors. To verify whether naloxone-induced cell apoptosis is correlated with ER stress, we examined the effect of naloxone on the expression of 
A

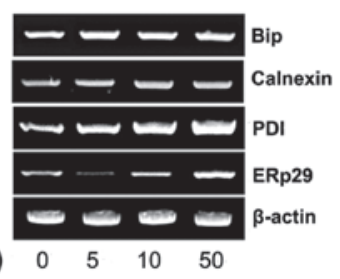

Dose $(\mu \mathrm{g}) \quad 0 \quad 5 \quad 10 \quad 50$
B

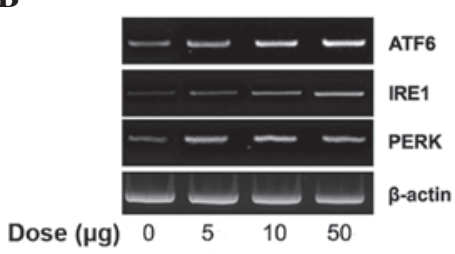

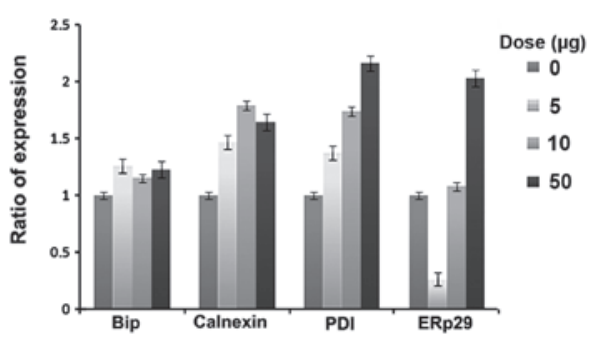

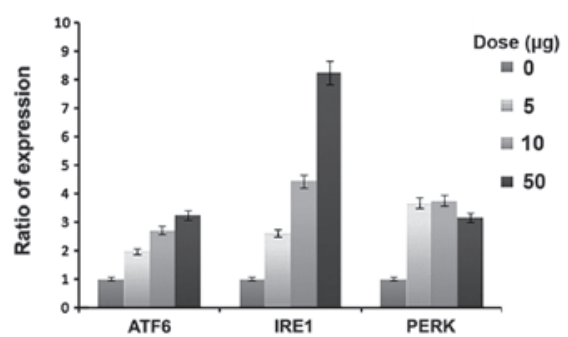

Figure 2. (A) Effect of naloxone on the gene expression of ER chaperones and (B) ER stress sensors. The PC12 cells were treated with the indicated concentrations of naloxone for $24 \mathrm{~h}$. Gene expression was determined by RT-PCR in the conditions described in Materials and methods. The experiments were performed three times and results represent the average. ER, endoplasmic reticulum; Bip, binding immunoglobulin protein; PDI, protein disulfide isomerase; ERp29, endoplasmic reticulum protein 29; ATF6, activating transcription factor 6; IRE1, inositol-requiring enzyme 1; PERK, double-stranded RNA-activated protein kinase-like ER kinase.

ER chaperones and ER stress sensors, namely Bip, ERp29 and PDI as well as the ER membrane chaperone calnexin. Naloxone dose dependently increased the expression of all ER chaperones (Fig. 2A). However, in general, the expression of Bip was relatively weak compared with others. Notably, ERp29 expression was markedly reduced at a low dose $(5 \mu \mathrm{g} / \mathrm{ml})$. The expression of the ER stress sensor also dose dependently increased (Fig. 2B) with naloxone using the similar experimental conditions. Particularly, the expression of IRE1 was relatively higher and, at the high dose $(50 \mu \mathrm{g} / \mathrm{ml})$, a 9-fold increase was noted.

Expression of ER stress signaling. We tested whether naloxone controls three ER-stress sensors (ATF6, PERK and IRE1) and cleaved ATF6, phosphorylated eIF2 $\alpha$ and spliced XBP1. With increasing concentrations of naloxone there was an increase in cleaved ATF6 (Fig. 3A), eIF2 $\alpha$ phosphorylation (Fig. 3B) and spliced XBP1 (Fig. 3C).

In vivo studies. To confirm the in vitro results, naloxone was injected into rat femurs and total RNA was isolated and subjected to RT-PCR using the conditions described in Materials and methods. The results of the in vivo studies, also demonstrate the same results as the in vitro studies. The animals treated with naloxone had increased expression of ER chaperones and ER stress sensors.

\section{Discussion}

In the present study, we documented that naloxone induces PC12 cell apoptosis in a dose-dependent manner $(5,10$ and $50 \mu \mathrm{g} / \mathrm{ml}$ ) using the MTT assay. However, the typical morphological hallmarks of apoptosis were not detected (data not shown). Doses higher than $50 \mu \mathrm{g} / \mathrm{ml}$ were not used due to cytotoxicity. The results demonstrate that naloxone induces apoptosis in PC12 cells in a dose-dependent manner (Fig. 1B).

To study whether PC12 cell apoptosis induced by naloxone is related to ER stress, we tested whether naloxone
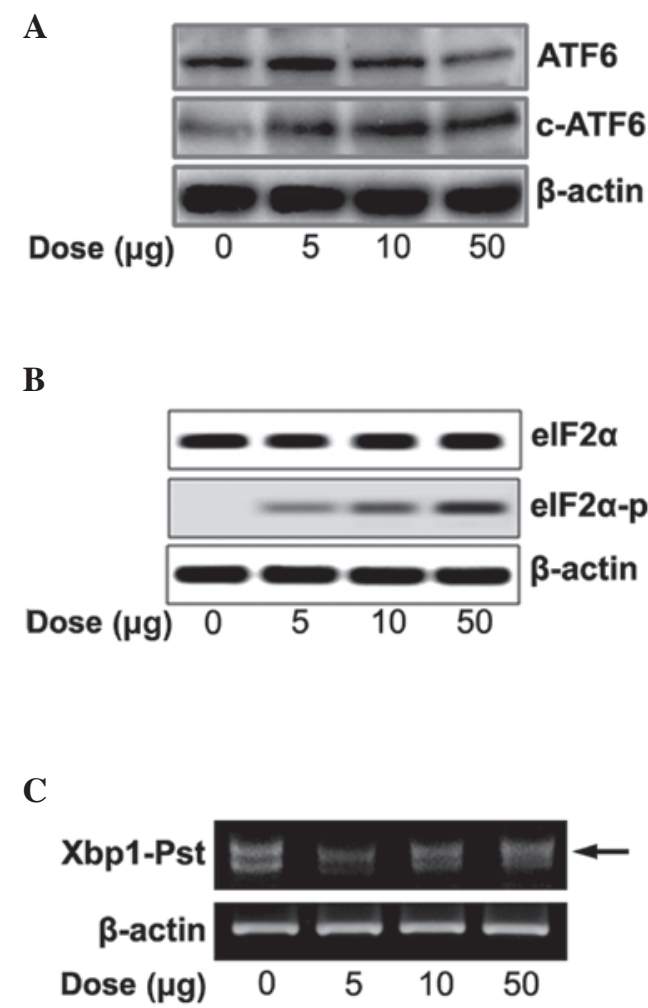

Figure 3. Activation of the ER stress signaling pathway by naloxone. (A) Cleavage of ATF6 protein; (B) phosphorylation of elF2 $\alpha$; (C) arrow indicates the product of Xbp1 mRNA splicing. The cells were treated with the indicated concentrations of naloxone for $24 \mathrm{~h}$. Cleavage and phosphorylation were assessed by western blotting. The product of Xbp1-Pst 1 digestion was analyzed by agarose gel electrophoresis. The experiments were performed three times and the results represent the average. c-ATF6, cleaved ATF6; elF $2 \alpha$, eukaryotic initiation factor 2 ; eIF $2 \alpha$-p, phosphorylation of eIF $2 \alpha$; Xbp1, X-box binding protein 1 .

induces the expression of ER chaperones and ER stress sensors. Bip, also known as GRP78, is one of the ER molecular chaperones located in the ER lumen that binds newly 
A
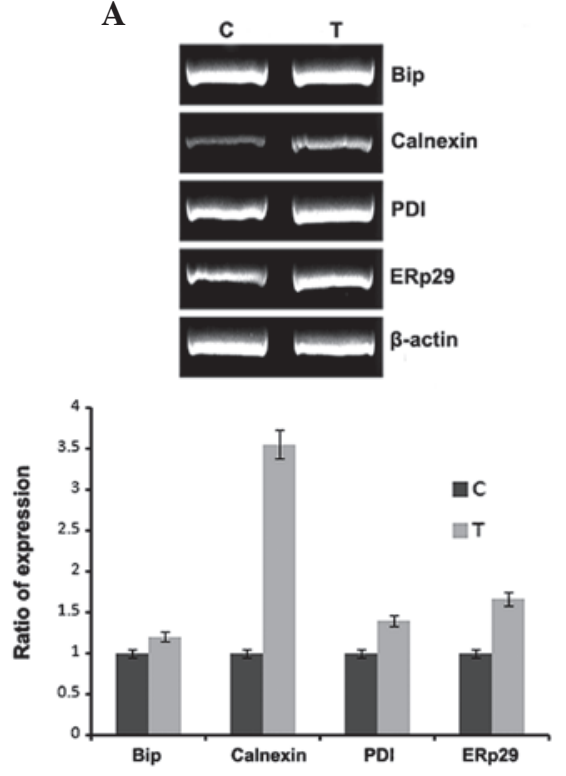
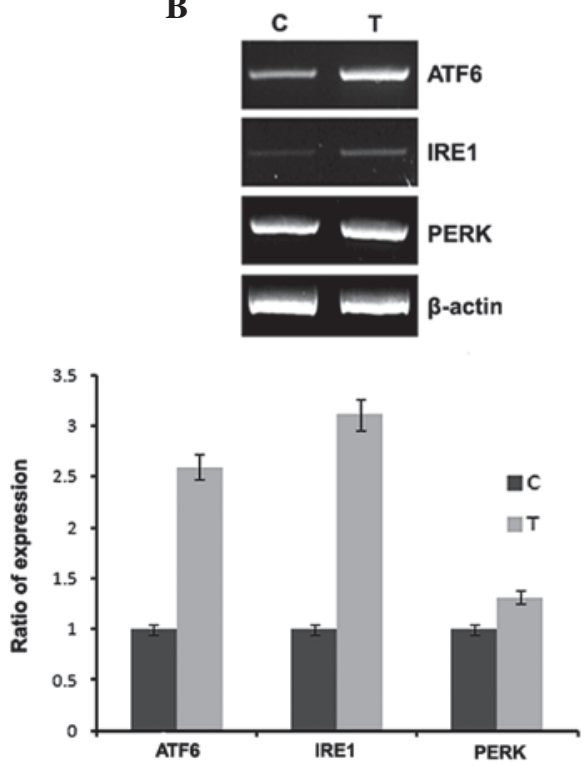

Figure 4. (A) Expression of ER chaperones and (B) ER stress sensors in vivo. Naloxone (1 mg/0.5 ml) was injected into rat femurs (Sprague-Dawley, average weight $130 \mathrm{~g}$ ). Following 1 day, skeletal muscle was obtained and purified to get the total RNA for RT-PCR. The experiments were performed three times and the results represent the average. Bip, binding immunoglobulin protein; PDI, protein disulfide isomerase; ERp29, endoplasmic reticulum protein 29; ATF6, activating transcription factor 6; IRE1, inositol-requiring enzyme 1; PERK, double-stranded RNA-activated protein kinase-like ER kinase.

synthesized proteins during translation and, under normal conditions, is bound to three ER stress sensors (13). PDI is an enzyme also located in the ER lumen that catalyzes the formation and breakage of disulfide bonds between cysteine residues within proteins (14). ERp29 demonstrates sequence similarity to PDI and it is important in the processing of secretory proteins within the ER (15). Calnexin is located in the ER membrane and its main function is quality control for unfolded or unassembled N-linked glycoproteins in the ER (16). As displayed in Fig. 2A, although the expression of Bip gradually increased following treatment with naloxone in a dose-dependent manner, compared with calnexin, the expression of PDI and ERp29 increased 2 -fold. The expression of ERp29 was specifically decreased at the low dose $(5 \mu \mathrm{g} / \mathrm{ml})$. The reason for this may be contention of the transcriptional factors biding to the ubiquitous enhancer occurring at the beginning of ER stress for ERp29. These results have been shown in other ERp29 experiments $(7,17)$. Under the same experimental conditions, naloxone dose dependently increased the gene expression of ER stress sensors (ATF6, IRE1 and PERK). When ER homeostasis is altered, the ER stress signaling pathway is mediated by activation of ER stress sensors. Although the expression of ER chaperones is increased 2-fold by naloxone stimulation, the expression of ER stress sensors is increased up to 9-fold (Fig. 2B). The stimulation of naloxone seems to be selective to the expression of ER stress sensors for maintaining normal cell physiology efficiently, rather than actively enhancing the expression of ER stress chaperones. It is suggested that the stimulation of naloxone markedly enhances the expression of ER stress sensors compared with those ER chaperones, as a cell-protection system through apoptosis.

We further tested whether naloxone stimulates ER stress signaling via the ER transmembrane proteins ATF6, IRE1 and PERK. Accumulation of un/misfolded proteins in the ER lumen triggers an ER stress signal pathway through ER stress sensors. It is known that, upon ER stress, releasing Bip from the ER luminal stress sensors cleaves ATF6 $\alpha$ and releases the transcription factors into the nucleus. Spliced XBP1 protein by IRE1 autophosphorylation finally acts as a transcription factor for induction of UPR target genes, and phosphorylation of eIF $2 \alpha$ by PERK autophosphorylation represses total protein synthesis (18). Naloxone concentration dependently increased the ER stress sensors and cleaved ATF6 $\alpha$ (Fig. 3A), phosphorylated eIF2 $\alpha$ (Fig. 3B) and increased spliced XBP1 (Fig. 3C), respectively. The results suggest that stimulation of naloxone directly regulates ER stress sensors as well as ER chaperones in a dose-dependent manner. In vivo experiments were conducted to confirm the in vitro data. The expression of ER chaperones and ER stress sensors are increased in a naloxone dose-dependent manner (Fig. 4). Although the expression of Bip, PDI and ERp29 are not significant, calnexin expression is stronger than its expression in vitro. The expression of ER stress sensors are almost the same in vitro and in vivo. However, it was confirmed that expression patterns, in vitro and in vivo, show a minor difference. However, in vitro expression of IRE1 and PERK increased 3.5 -fold and 1.5-fold, while in vivo the expression increased 9-fold and 4-fold, respectively.

In summary, the present study, to the best of our knowledge, is the first to demonstrate that ER chaperones (Bip, calnexin, PDI and ERp29) and ER stress sensors (ATF6, IRE1 and PERK) were upregulated by naloxone in a dose-dependent manner. Our findings suggest that ER stress may be involved in naloxone-induced PC12 cell apoptosis, which may provide new insight into the possible role of naloxone in ER stress. This may aid the development of novel drugs for ER stressassociated diseases, including diabetes, inflammation and neurodegenerative disorders, including Alzheimer's disease and Parkinson's disease. 


\section{Acknowledgements}

This study was supported by the Basic Science Research Program through the National Research Foundation of Korea (NRF) and was funded by the Ministry of Education, Science and Technology (2010-0009806).

\section{References}

1. Ashton $\mathrm{H}$ and Hassan Z: Best evidence topic report. Intranasal naloxone in suspected opioid overdose. Emerg Med J 23: 221-223, 2006.

2. Handal KA, Schauben JL and Salamone FR: Naloxone. Ann Emerg Med 12: 438-445, 1983.

3. Krizanac-Bengez L, Boranić M, Testa NG and Kardum I: Naloxone interferes with granulocytopoiesis in long-term cultures of mouse bone marrow; buffering by the stromal layer Res Exp Med (Berl) 194: 375-382, 1994.

4. Liu SL, Li YH, Shi GY, et al: A novel inhibitory effect of naloxone on macrophage activation and atherosclerosis formation in mice. J Am Coll Cardiol 48: 1871-1879, 2006.

5. Beverley CL, Higgins PJ and Borenfreund E: The effect of methadone and naloxone on cultured rat liver cells. Exp Cell Biol 52: 170-175. 1984.

6. Almela P, Milanés MV and Laorden ML: Activation of the ERK signalling pathway contributes to the adaptive changes in rat hearts during naloxone-induced morphine withdrawal. Br J Pharmacol 151: 787-797, 2007.

7. Almela P, Martínez-Laorden E, Atucha NM, et al: Naloxoneprecipitated morphine withdrawal evokes phosphorylation of heat shock protein 27 in rat heart through extracellular signalregulated kinase. J Mol Cell Cardiol 51: 129-139, 2011.
8. Venihaki M, Gravanis A and Margioris AN: Opioids inhibit dopamine secretion from PC12 rat pheochromocytoma cells in a naloxone-reversible manner. Life Sci 58: 75-82, 1996.

9. Claessen JH, Kundrat L and Ploegh HL: Protein quality control in the ER: balancing the ubiquitin checkbook. Trends Cell Biol 22: 22-32, 2012.

10. Ni M and Lee AS: ER chaperones in mammalian development and human diseases. FEBS Lett 581: 3641-3651, 2007.

11. Back SH and Kaufman RJ: Endoplasmic reticulum stress and type 2 diabetes. Annu Rev Biochem 81: 767-793, 2012.

12. Jin W, Lee NM, Loh HH and Thayer SA: Opioids mobilize calcium from inositol 1,4,5-trisphosphate-sensitive stores in NG108-15 cells. J Neurosci 14: 1920-1929, 1994.

13. Otero JH, Lizák B and Hendershot LM: Life and death of a BiP substrate. Semin Cell Dev Biol 21: 472-478, 2010.

14. Wilkinson B and Gilbert HF: Protein disulfide isomerase. Biochim Biophys Acta 1699: 35-44, 2004.

15. Zhang D and Richardson DR: Endoplasmic reticulum protein 29 (ERp29): An emerging role in cancer. Int J Biochem Cell Biol 43: 33-36, 2011.

16. Chevet E, Smirle J, Cameron PH, et al: Calnexin phosphorylation: linking cytoplasmic signalling to endoplasmic reticulum lumenal functions. Semin Cell Dev Biol 21: 486-490, 2010

17. Lee KR, Kim SW, Kim YK, et al: Silkworm Hemolymph DownRegulates the Expression of Endoplasmic Reticulum Chaperones under Radiation-Irradiation. Int J Mol Sci 12: 4456-4464, 2011.

18. Shore GC, Papa FR and Oakes SA: Signaling cell death from the endoplasmic reticulum stress response. Curr Opin Cell Biol 23: 143-149, 2011. 